\title{
Measurement of the energy distribution of trapped and free electrons in a current-free double layer
}

\author{
K. Takahashi, ${ }^{\text {a) }}$ C. Charles, and R. W. Boswell \\ Space Plasma, Power and Propulsion, Research School of Physical Sciences and Engineering, \\ The Australian National University, Canberra ACT 0200, Australia \\ T. Kaneko and R. Hatakeyama \\ Department of Electronic Engineering, Tohoku University, Sendai 980-8579, Japan
}

(Received 16 August 2007; accepted 8 October 2007; published online 21 November 2007)

\begin{abstract}
In the high potential plasma, upstream of the double layer, the measured electron energy distribution function (EEDF) shows a very clear change in slope at energies $\left(\varepsilon_{\text {break }}\right)$ corresponding to the double layer potential drop. Electrons with lower energy are Maxwellian with a temperature of $8 \mathrm{eV}$, whereas those with higher energy have a temperature of $5 \mathrm{eV}$. The EEDF in the downstream plasma has a temperature of $5 \mathrm{eV}$. Over the range of pressures wherein the double layer and accelerated ion beam are detected by analysis of a retarding field energy analyzer, the strength of the double layer corresponds to the energy where the slope changes in the EEDF $\left(\varepsilon_{\text {break }}\right)$. We deduce that the downstream electrons come from upstream electrons that have sufficient energy to overcome the potential of the double layer, and that only a single upstream plasma source is required to maintain this phenomenon. (C) 2007 American Institute of Physics. [DOI: 10.1063/1.2803763]
\end{abstract}

High energy charged particles in space are thought to be accelerated by mechanisms such as proposed by Fermi, by waves, and more recently, by electric double layers (DLs). ${ }^{1-3}$ Although some experimental data obtained from probes on satellites are available, they are rarely sufficient to fully develop self-consistent models of such space DLs and assumptions on the form of the accelerated and trapped particle distribution functions have to be made. Perkins and Sun predicted the existence of current-free double layer solutions, ${ }^{4}$ and soon after their prediction, current-driven laboratory double layers were set up in a current-free "mode," firming the prediction. More recently, a new class of currentfree double layers were experimentally found in expanding plasmas. $^{8-10}$

In this Brief Communication, we provide experimental measurements of the EEDF upstream and downstream of a current-free double layer: the upstream EEDF shows a Maxwellian distribution out to an $\varepsilon_{\text {break }}$ and a depleted distribution for higher energies, whereas the downstream EEDF mirrors very closely the depleted upstream population. This suggests that the upstream plasma fully supports the double layer and the downstream plasma rather than the double layer being the interface between two separate and distinct plasmas. We also show that the earlier, preliminary, measurements of an upstream electron beam with a positive slope to the EEDF ${ }^{11}$ were likely the result of rf interference as the measurements could not be convincingly reproduced. However, the present results do show that the downstream electrons do appear to be accelerated by the double layer into the upstream plasma and hence are a beam, although the system would not be unstable according to the Penrose criterion. ${ }^{12}$

\footnotetext{
${ }^{a}$ Permanent address: Department of Electronic Engineering, Tohoku University. Present address: Department of Electrical and Electronic Engineering, Iwate University. Electronic mail: kazunori@iwate-u.ac.jp.
}

Non-Maxwellian features have been observed in many rf plasmas. In particular, the depletion of high energy electrons has been attributed to inelastic collisions at comparatively high gas pressure ${ }^{13,14}$ with the lower energy limit $\left(\varepsilon_{\text {break }}\right)$ observed in the EEDF being associated with either the excitation or ionization energies. At lower gas pressure of greater relevance here, $\varepsilon_{\text {break }}$ was shown to correlate well with the magnitude of the wall sheath. ${ }^{15}$ A recent particle-in-cell (PIC) simulation ${ }^{16}$ reported that electrons trapped in a low pressure discharge by the wall sheaths possess an EEDF having a Maxwellian group of trapped electrons, and beyond an $\varepsilon_{\text {break }}$, a depleted group of high energy electrons that escape to the (absorbing) walls. The $\varepsilon_{\text {break }}$ was found to be close to the sheath potential. More pertinent to the present experiment, further results from a simulation of a current-free DL in an expanding plasma show an EEDF in the upstream plasma having a trapped group of Maxwellian electrons up to an $\varepsilon_{\text {break }}$ and then a depleted group of high energy electrons. In the downstream plasma the EEDF appears to resemble the depleted high energy upstream population. By plotting $\varepsilon_{\text {break }}$ and the local plasma potential as a function of axial distance, the authors were able to clearly show the presence of the double layer. ${ }^{16}$

The measurement of the EEDF is difficult, especially in the presence of radio frequency electric fields in the plasma that can severely distort the results. For the present work a cylindrical, rf-compensated, Langmuir probe is used to measure the EEDF. The design of the probe follows Ref. 17 with an exposed nickel wire $3 \mathrm{~mm}$ long and $0.25 \mathrm{~mm}$ in diameter connected through a glass to metal seal to five rf choke coils mounted in series to filter any plasma potential oscillation at the fundamental $(13.56 \mathrm{MHz})$ or second-harmonic (27.12 MHz) frequencies. A reference electrode is wound around the outside of the glass sealing tube from which the probe protrudes, enters through this glass tube, and is con- 


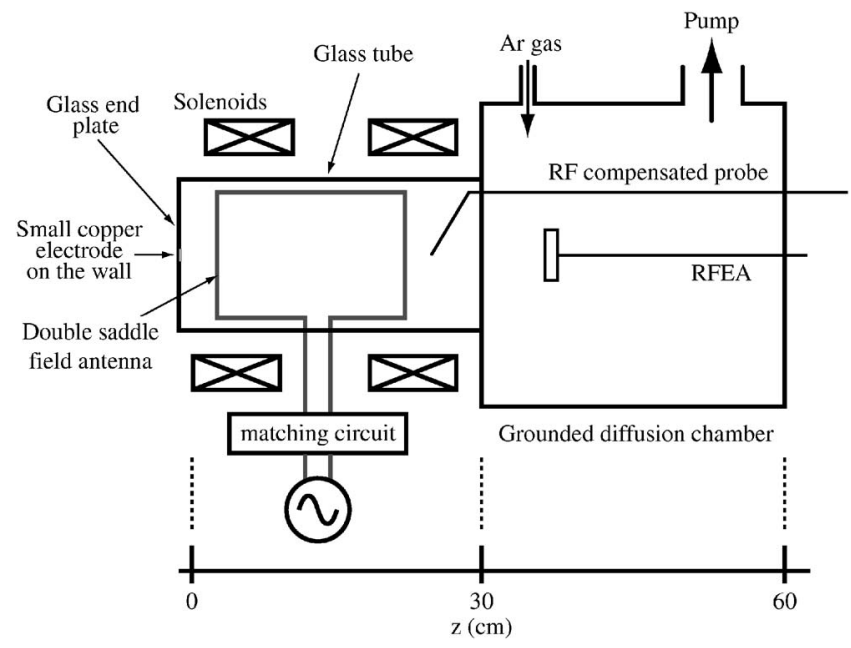

FIG. 1. Schematic diagram of "Chi-Kung" helicon reactor.

nected to the probe wire through a $4.7 \mathrm{nF}$ capacitor. Outside the plasma the current signal is passed through a $100 \Omega$ resistor and then is connected to an earth isolated bipolar voltage source that is swept at $10 \mathrm{~Hz}$ from -120 to $+120 \mathrm{~V}$. The voltage signal from the resistor is fed through an isolation amplifier to two resonant circuits tuned to $800 \mathrm{~Hz}$ that act both as rejecting filters for low frequency plasma instabilities ${ }^{18}$ and as differentiators. ${ }^{19}$ Finally, the signals are digitized at $50 \mathrm{kHz}$ and 14 bits, and then passed into a LABVIEW program for display on a computer. This system allows current-voltage curves to be measured over about three orders of magnitude of current before random and/or digitizing noise becomes important. The second derivative of the current voltage trace contains the EEDF and the local plasma potential $\left(V_{p}\right)$, the latter being taken as the zero crossing of the curve and the EEDF being the section of the curve to the left of $V_{p}$. This is because the electrons in the plasma see the local plasma potential as a zero reference for the EEDF and the sweeping voltage of the probe uncovers more and more of the EEDF as it approaches $V_{p}$ from below. Hence, the zero reference of the local EEDF actually represents the local $V_{p}$.

Experiments are performed in the "Chi-Kung" helicon reactor shown in Fig. 1 and previously described in Refs. 8 and 11. Briefly, a helicon plasma source consisting of a $13.7 \mathrm{~cm}$ inner diameter, $31 \mathrm{~cm}$ long cylindrical glass tube is connected contiguously to a grounded diffusion chamber of $30 \mathrm{~cm}$ in length, and $32 \mathrm{~cm}$ in diameter. A $13.56 \mathrm{MHz}$ generator supplies $250 \mathrm{~W}$ to the double-saddle rf antenna surrounding the source tube that contains argon at $P_{\mathrm{Ar}}$ $=0.2-2 \mathrm{mTorr}$. Two solenoids situated around the source are used to create an expanding magnetic field with a maximum of about $130 \mathrm{G}$ in the source center, decreasing to a few tens of Gauss in the diffusion chamber. ${ }^{20}$ Under these conditions, a DL is created near the open end of the helicon source $(z \simeq 25 \mathrm{~cm})$, where we define $z=30 \mathrm{~cm}$ as the boundary between the source and the diffusion chamber. This is verified by measuring a supersonic ion beam using a retarding field energy analyzer (RFEA) at $z=37 \mathrm{~cm}$ at the same time as the Langmuir probe biased to collect electrons at $100 \mathrm{~V}$ positive is inserted in the upstream area. The DC potential of the

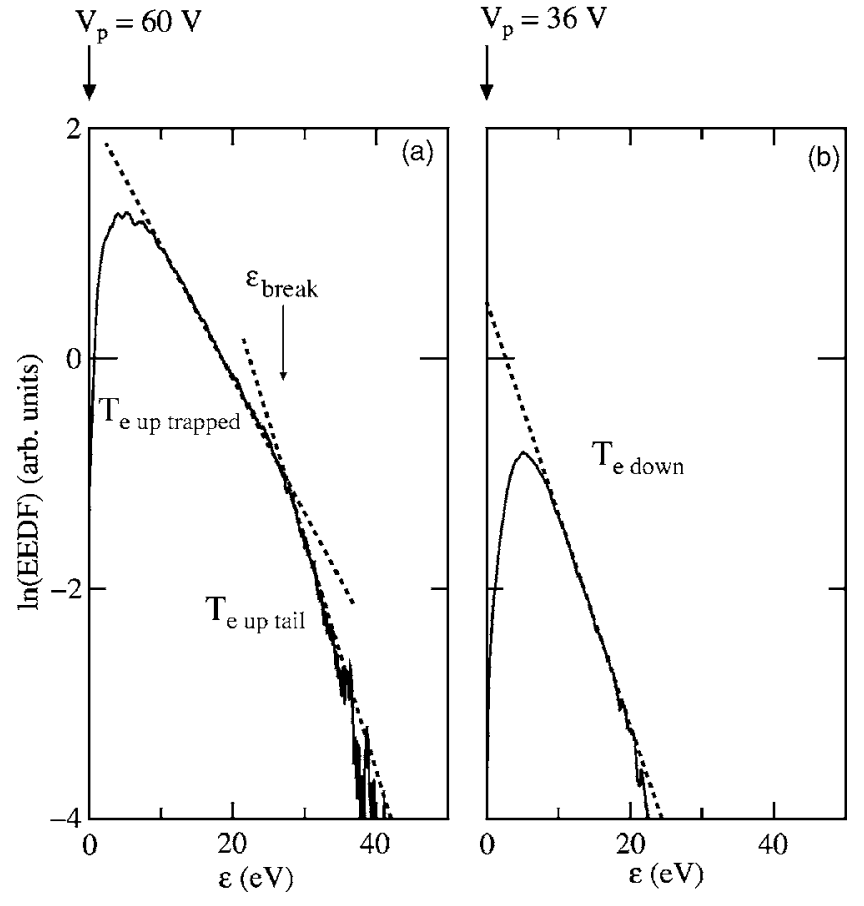

FIG. 2. Natural logarithm plot of electron energy distribution functions $($ EEDF) in (a) the upstream area $(z=17 \mathrm{~cm})$ and (b) the downstream area $(z=36 \mathrm{~cm})$ for $P_{\mathrm{Ar}}=0.3 \mathrm{mT}$ orr. The dashed lines show the tangential lines giving each temperature $T_{e}$ up trapped $(\sim 8 \mathrm{eV}), T_{e}$ up tail $(\sim 5 \mathrm{eV})$, and $T_{e \text { down }}$ $(\sim 5 \mathrm{eV})$. The break energy of the EEDF depletion at $27 \mathrm{eV}$ is defined as $\varepsilon_{\text {break }}$ as indicated in Fig. 2(a).

floating wall in the upstream (source) plasma is measured using a thin disk of $8 \mathrm{~mm}$ diameter set on the inside of the glass end plate. The disk is connected to an oscilloscope (input impedance: $1 \mathrm{M} \Omega$ ) through a $50 \mathrm{M} \Omega$ resistor and the measured voltage taken to be the wall potential.

The upstream EEDF at $z=17 \mathrm{~cm}$ is plotted in Fig. 2(a), with $\varepsilon=0$ corresponding to the local $V_{p}$ of $60 \mathrm{~V}$, and shows a very clear $\varepsilon_{\text {break }}$ in the slope at about $27 \mathrm{eV}$, which corresponds very closely to the potential drop of the DL measured with the RFEA. Below $\varepsilon_{\text {break }}$, the slope of the EEDF yields a temperature of $\sim 8 \mathrm{eV}$, whereas above $\varepsilon_{\text {break }}$, the temperature is $\sim 5 \mathrm{eV}$. Figure 2(b) shows the downstream EEDF at $z$ $=36 \mathrm{~cm}$ (local $V_{p}$ of $36 \mathrm{~V}$ ) on the same (ordinate) current scale as Fig. 2(a). The relative differences in the maximum of the two graphs represents a density difference between the upstream and downstream plasmas of about a factor of 7, which also agrees well with the RFEA measurements presented previously. ${ }^{8}$ Downstream, the EEDF is fitted with a temperature of $\sim 5 \mathrm{eV}$, matching that of the depleted upstream population. The difference in local $V_{p}$ for Figs. 2(a) and 2(b) also matches quite well the $\varepsilon_{\text {break }}$ given the approximate nature of measuring both the plasma potential and the $\varepsilon_{\text {break }}$. The signal-to-noise ratio is not sufficient to get adequate information for downstream electron energies greater than $20 \mathrm{eV}$, although a depletion of the EEDF from a Maxwellian due to the escape of fast electrons to the grounded (right-hand) wall would be expected at the energy corresponding to the local plasma potential, or right-hand sheath potential in this case. It seems quite clear that the depleted group of upstream electrons with energies greater than $\varepsilon_{\text {break }}$ 


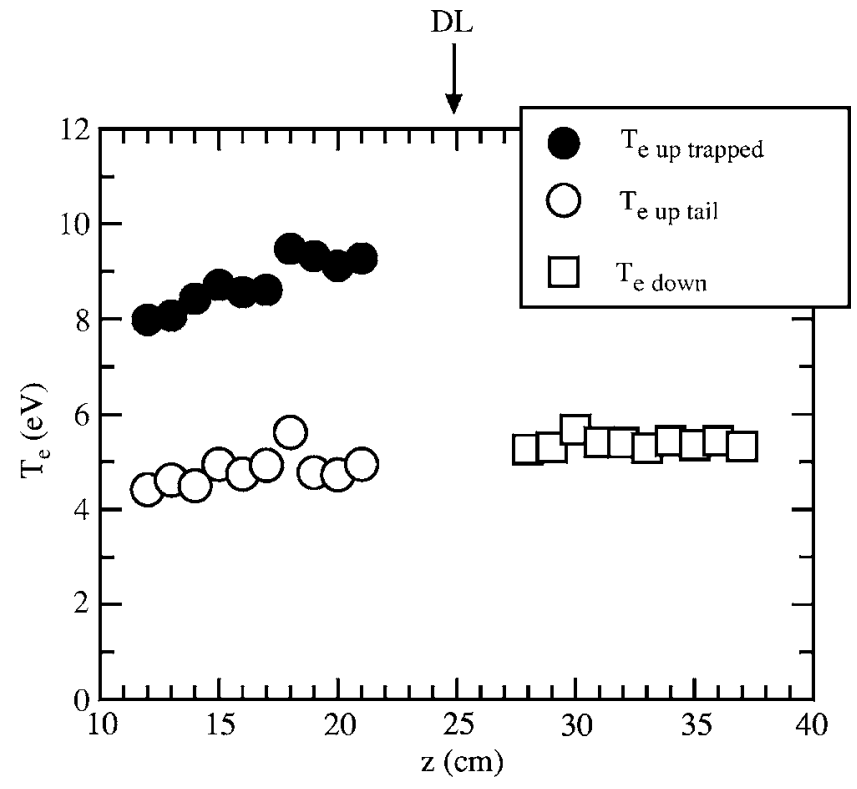

FIG. 3. Axial profiles of electron temperatures $T_{e}$ up trapped (closed circle), $T_{e}$ up tail (open circle), and $T_{e \text { down }}$ (open square) for $P_{\mathrm{Ar}}=0.3 \mathrm{mTorr}$.

match in both density and temperature very well with the downstream group of electrons, suggesting very strongly that they are the same group of electrons that move from upstream to downstream by climbing the double layer potential of magnitude $\varepsilon_{\text {break }}$.

The axial variation of the three electron temperatures (upstream trapped, upstream tail, and downstream) are shown in Fig. 3, where closed circle, open circle, and open square are $T_{e}$ up trapped, $T_{e}$ up tail , and $T_{e \text { down }}$, respectively. The temperatures are fairly constant along the main axis of the experiment and the relation $T_{e}$ up tail $\simeq T_{e \text { down }}$ is clearly seen, which further suggests the proposition that the depleted high energy group of electrons in the upstream area is moving downstream by climbing the potential drop of the DL. It seems likely that, in this way, the kinetic energy of the electrons is given up to charging the double layer potential. No results for the electron temperature are shown for the region 22 to $27 \mathrm{~cm}$ as the EEDF appeared to have a Druyvesteyn form without any part resembling a Maxwellian. This is probably because we are trying to measure the EEDF in a very strong electric field, where it is expected that this type of distribution would be detected. ${ }^{21}$ This behavior near the DL has also been reported in past studies. ${ }^{22,23}$ In this area, it is also difficult to use the double differentiated currentvoltage curve when trying to find the plasma potential. However, $8 \mathrm{~cm}$ upstream and $11 \mathrm{~cm}$ downstream of the DL, the plasma potential measurements made by the RFEA and by the rf-compensated Langmuir probe agree $\left(V_{p}=60-70 \mathrm{~V}\right.$ at $z=17 \mathrm{~cm}$ and $V_{p} \simeq 30-36 \mathrm{~V}$ at $\left.z=36 \mathrm{~cm}\right)$ to within experimental confidence levels. Some experiments were also conducted with an emissive probe which yielded plasma potential results in agreement with other methods downstream, but the data could not be interpreted in the region around the double layer.

In order to have more confidence in the correlation between the break point energy and the magnitude of the

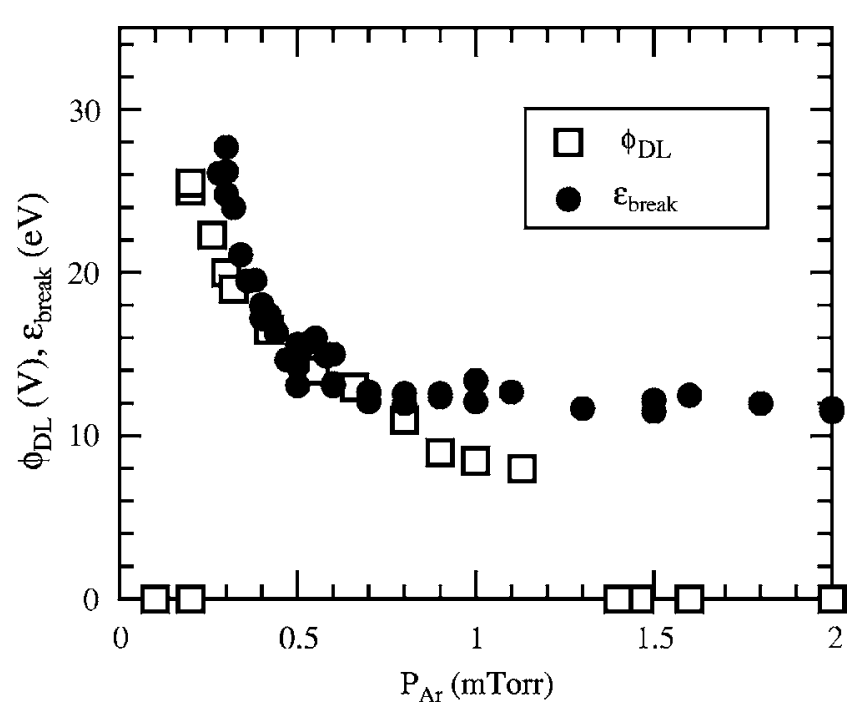

FIG. 4. The break energy $\varepsilon_{\text {break }}$ (closed circle) and the potential drop $\phi_{\mathrm{DL}}$ of the DL (open square) as a function of argon gas pressure $P_{\mathrm{Ar}}$. The experimental data on $\phi_{\mathrm{DL}}$ are from Refs. 24 and 25.

double layer, it would be useful to be able to change these parameters over a reasonable range. In earlier work it has been shown ${ }^{24,25}$ that the potential drop $\phi_{\mathrm{DL}}$ of the DL is strongly dependent on the gas pressure $P_{\mathrm{Ar}}$. The break energy $\varepsilon_{\text {break }}$ as a function of $P_{\mathrm{Ar}}$ is plotted in Fig. 4 as closed circles together with the potential drop $\phi_{\mathrm{DL}}$ (open square), where the experimental results on $\phi_{\mathrm{DL}}$ are from Refs. 24 and 25 . The DL potential drop is found to track the break energy very well for pressures of $P_{\mathrm{Ar}} \simeq 0.2-1.2$ mTorr with a small divergence at the high pressure end. For pressures greater than about 1.2 mTorr, a $\varepsilon_{\text {break }}$ is still observed and remains constant at about $12 \mathrm{eV}$. This break energy possibly originates from inelastic collisions described in Refs. 13 and 14, where the excitation energy of argon is $11.55 \mathrm{eV}$, or it may reflect the magnitude of the sheath on the right-hand wall. Clearly there are a number of factors contributing to the value of $\varepsilon_{\text {break }}$ at the higher pressures, which could explain the small divergence from $\phi_{\mathrm{DL}}$ around $1 \mathrm{mTorr}$.

Before we conclude that the break energy is related to the DL, the electrons escaping to the floating wall on the left side should be discussed. The local plasma potential in the upstream area is observed to be about $60-70 \mathrm{~V}$ for $P_{\mathrm{Ar}}$ $=0.3 \mathrm{mTorr}$ and the potential measured by the small disk embedded on the left-side wall is about $16 \mathrm{~V}$. Hence, the potential drop $\phi_{\text {left }}$ of the left-side sheath is about $44-54 \mathrm{~V}$ and as $\phi_{\text {left }}>\phi_{\mathrm{DL}}\left(\phi_{\mathrm{DL}}=24-30 \mathrm{~V}\right)$, the break point is clearly not related to electrons escaping over the left-side sheath.

The electron energy distribution function (EEDF) in a current-free double layer with an upstream helicon rf source has been experimentally investigated. Upstream, the EEDF is Maxwellian up to an energy determined by the DL potential and shows a depletion for higher energies. In the downstream region the EEDF is approximately Maxwellian and very closely resembles the shape and magnitude of the depleted upstream population. The break energy of the upstream 
EEDF clearly tracks the potential drop of DL as the pressure is varied.

These results do not show a beam of electrons arriving from the downstream plasma, in the sense that there is nowhere a positive slope in the EEDF. It does show that upstream, the depleted tail electrons clearly arrive from downstream and thus, on their own, can be considered an accelerated group of downstream electrons entering the upstream population. In this sense, the experimental results are in excellent agreement with the kinetic PIC simulations ${ }^{16}$ that show EEDFs having precisely the same form. The fluid model $^{24,25}$ also requires an accelerated group of beam electrons arriving from downstream to satisfy the charge conditions for the existence of the double layer but uses a fluid definition of equal electron temperature both upstream and downstream. Hence, the present results, while not showing a classical beam of electrons, do show elements that agree with both the simulation and the fluid models and strongly suggest that both are presently too simplistic to adequately model the details of the experiment. On the other hand, it should be mentioned that the fluid model shows quantitative agreement with the experiment over a wide parameter range and thus is able to usefully predict the behavior of the double layer. However, the details of the kinetic behavior need to be incorporated, and this is the next challenge for the theory.

Electrons with energies lower than $\phi_{\mathrm{DL}}$ in the upstream area are electrostatically trapped between the left-side floating wall and the DL. These electrons are heated by the rf electromagnetic fields for the helicon source and have a relatively high temperature. Higher energy electrons can overcome the electrostatic potential barrier of the DL and propagate into the downstream area while being decelerated by the DL. Those electrons that are not scattered would subsequently be reflected by the sheath on the grounded right-side wall and return to the upstream area, regaining the energy they had previously lost to the DL structure and forming a half-Maxwellian beam of accelerated electrons entering the trapped upstream population. The effects of the rf heating on these electrons is much smaller than that on the trapped electrons, as they spend considerably less time in the heating region compared to the time necessary to move between the two regions. Hence, it would seem that there is a single source of plasma for the system, with ions being lost to the downstream region by acceleration through the DL and a depleted group of electrons created upstream overcoming the DL potential and propagating into the downstream region.

The authors are indebted to Peter Alexander for his technical assistance. This work was supported by Tohoku University 21st COE program, System Construction of GlobalNetwork Oriented Information Electronics, and by the Japan Society for the Promotion of Science for Young Scientists. It was also supported by a Visiting Fellowship of the Research School of Physical Sciences and Engineering. K.T. wishes to thank all the members of the SP3 group of the Plasma Research Laboratory at ANU for their hospitality during his three-month stay, and Dr. C. Charles, Professor R. W. Boswell, and Professor R. Hatakeyama for supervising this project.

${ }^{1}$ A. C. Williams, M. C. Weisskopf, R. F. Elsner, W. Darbro, and P. G. Sutherland, Astrophys. J. 305, 759 (1986).

${ }^{2}$ N. Borisov and U. Mall, J. Plasma Phys. 67, 277 (2002).

${ }^{3}$ R. W. Boswell, E. Marsch, and C. Charles, Astrophys. J. Lett. 640, L199 (2006).

${ }^{4}$ F. W. Perkins and Y. C. Sun, Phys. Rev. Lett. 46, 115 (1981).

${ }^{5}$ C. Chan, N. Hershkowitz, and G. L. Payne, Phys. Lett. 83A, 328 (1981).

${ }^{6}$ R. Hatakeyama, Y. Suzuki, and N. Sato, Phys. Rev. Lett. 50, 1203 (1983).

${ }^{7}$ G. Hairapetian and R. L. Stenzel, Phys. Rev. Lett. 65, 175 (1990).

${ }^{8}$ C. Charles and R. W. Boswell, Appl. Phys. Lett. 82, 1356 (2003).

${ }^{9}$ S. A. Cohen, N. S. Siefert, S. Stange, R. F. Boivin, E. E. Scime, and F. M. Levinton, Phys. Plasmas 10, 2593 (2003).

${ }^{10}$ X. Sun, A. M. Keesee, C. Biloiu, E. E. Scime, A. Meige, C. Charles, and R. W. Boswell, Phys. Rev. Lett. 95, 025004 (2005).

${ }^{11}$ C. Charles and R. W. Boswell, Phys. Plasmas 11, 1706 (2004).

${ }^{12}$ O. Penrose, Phys. Fluids 3, 2 (1960).

${ }^{13}$ U. Kortshagen, I. Pukropski, and M. Zethoff, J. Appl. Phys. 76, 2048 (1994).

${ }^{14}$ V. A. Godyak, R. B. Piejak, and B. M. Alexandrovich, Plasma Sources Sci. Technol. 4, 332 (1995).

${ }^{15}$ V. A. Godyak, R. B. Piejak, and B. M. Alexandrovich, Plasma Sources Sci. Technol. 11, 525 (2002).

${ }^{16}$ A. Meige and R. W. Boswell, Phys. Plasmas 13, 092104 (2006).

${ }^{17}$ I. D. Sudit and F. F. Chen, Plasma Sources Sci. Technol. 3, 162 (1994).

${ }^{18}$ A. Aanesland, C. Charles, M. A. Lieberman, and R. W. Boswell, Phys. Rev. Lett. 97, 075003 (2006).

${ }^{19}$ K. F. Schoenberg, Rev. Sci. Instrum. 49, 1377 (1978).

${ }^{20}$ C. Charles, Phys. Plasmas 12, 044508 (2005).

${ }^{21}$ W. P. S. Tan, J. Phys. D 6, 1206 (1973).

${ }^{22}$ R. Schrittwieser, I. Axnäs, T. Carpenter, and S. Torvén, IEEE Trans. Plasma Sci. 20, 607 (1992).

${ }^{23}$ S. Torvén, J. Phys. D 15, 1943 (1982).

${ }^{24}$ M. A. Lieberman and C. Charles, Phys. Rev. Lett. 97, 045003 (2006).

${ }^{25}$ M. A. Lieberman, C. Charles, and R. W. Boswell, J. Phys. D 39, 3294 (2006). 